\title{
Treatment of aneurysms in patients with moyamoya disease: a 10-year single-center experience
}

\author{
*Wei Ni, MD, PhD, Hanqiang Jiang, MD, PhD, Bin Xu, MD, PhD, Yu Lei, MD, Heng Yang, MD, \\ Jiabin Su, MD, Yuxiang Gu, MD, PhD, and Ying Mao, MD, PhD \\ Division of Cerebrovascular Surgery and Interventional Neuroradiology, Department of Neurosurgery, Huashan Hospital, Fudan \\ University, Shanghai, People's Republic of China
}

\begin{abstract}
OBJECTIVE Moyamoya disease (MMD) is occasionally accompanied by intracranial aneurysms. The purpose of this study was to delineate the efficacy of the authors' current surgical strategy in the management of MMD-associated aneurysms of different types.

METHODS Between January 2007 and March 2016, a consecutive cohort of 34 patients with 36 MMD-associated aneurysms was enrolled in this prospective single-center cohort study. The lesions were classified as peripheral (17 aneurysms) or main trunk aneurysms ( 13 in the anterior circulation and 6 in the posterior circulation). For the peripheral aneurysms, revascularization with or without endovascular treatment was suggested. For the main trunk aneurysms, revascularization alone, revascularization with aneurysm clipping, or revascularization with aneurysm embolization were used, depending on the location of the aneurysms.

RESULTS Of the peripheral aneurysms, 4 were treated endovascularly with staged revascularization, and 13 were treated solely with cerebral revascularization. Of the 13 main trunk aneurysms in the anterior circulation, 10 were clipped followed by revascularization, and 3 were coiled followed by staged cerebral revascularization. Of the 6 main trunk aneurysms in the posterior circulation, 4 underwent endovascular coiling and 2 were treated solely with revascularization. One patient died of contralateral intracerebral hemorrhage 6 months after the operation. No other patients suffered recurrent intracranial hemorrhage, cerebral ischemia, or aneurysm rupture. An angiographic follow-up study showed that all the bypass grafts were patent. Complete occlusion was achieved in all 21 aneurysms that were clipped or embolized. Of the remaining 15 aneurysms that were not directly treated, 12 of 13 peripheral aneurysms were obliterated during the follow-up, whereas 1 remained stable; 1 of 2 posterior main trunk aneurysms remained stable, and the other became smaller.
\end{abstract}

CONCLUSIONS The authors' current treatment strategy may benefit patients with MMD-associated aneurysms. https://thejns.org/doi/abs/10.3171/2017.3.JNS162290

KEY WORDS moyamoya disease; aneurysm; revascularization; treatment strategy; vascular disorders

$\mathrm{M}$ OYAMOYA disease (MMD) is an uncommon cerebrovascular disease characterized by an unusual vascular network at the base of the brain caused progressive stenosis or occlusion of the supraclinoid internal carotid artery (ICA) and its main branches within the circle of Willis. ${ }^{15}$ Occasionally MMD is accompanied by cerebral aneurysms. The incidence of intracranial aneurysms in patients with MMD has been estimated to be
$3 \%-14 \%$ in adults. ${ }^{29}$ These aneurysms are classified as peripheral aneurysms and major artery aneurysms ${ }^{23}$ Peripheral aneurysms are caused by rupture of the pathological moyamoya vessels or distal dilated choroidal arteries. The major aneurysms are almost saccular aneurysms that arise from the circle of Willis and lead to subarachnoid hemorrhage (SAH).

Although MMD with aneurysms is rarely encountered,

ABBREVIATIONS $\mathrm{ACA}=$ anterior cerebral artery; $\mathrm{AChA}=$ anterior choroidal artery; $\mathrm{ACo} A=$ anterior communicating artery; $\mathrm{BA}=$ basilar artery; $\mathrm{DSA}=$ digital subtraction angiography; $E C A$ = external carotid artery; $E D M S=$ encephaloduromyosynangiosis; $I C A=$ internal carotid artery; $I C H=$ intracerebral hemorrhage; $I V H=$ intraventricular hemorrhage; $L S A=$ lenticulostriate artery; $M C A=$ middle cerebral artery; $M M D=$ moyamoya disease; $\mathrm{mRS}=$ modified Rankin Scale; OphA = ophthalmic artery; $P C A=$ posterior cerebral artery; PChA = posterior choroidal artery; SAH = subarachnoid hemorrhage; STA-MCA = superficial temporal artery-middle cerebral artery.

SUBMITTED September 4, 2016. ACCEPTED March 6, 2017.

INCLUDE WHEN CITING Published online August 25, 2017; DOI: 10.3171/2017.3.JNS162290.

* Drs. Ni and Jiang contributed equally to this work. 
the prognosis is relatively poor. ${ }^{6,11}$ The peripheral aneurysms, usually originating from distal fragile arteries, are more likely to rerupture in patients with hemorrhagic presentation. ${ }^{6,27}$ The main trunk aneurysms, which are formed due to hemodynamic disturbance, have a higher risk of spontaneous progression or rupture, resulting in fatal SAH. Hence, early intervention for the aneurysms in patients with MMD should be considered.

In the past decades, the optimal strategy for the treatment of MMD-associated aneurysms has remained controversial. For peripheral aneurysms, surgical clipping is complicated because of the difficult location of the aneurysms. Endovascular treatment is sometimes challenging due to the tortuous approach of the parent artery. Cerebral revascularization can lead to the spontaneous disappearance of peripheral aneurysms but still needs further investigation. ${ }^{7,22}$ For main trunk aneurysms, there has been no unified treatment to date. Some experts favor surgical intervention for these aneurysms to reduce the possibility of SAH. ${ }^{12,31}$ However, some aneurysms in the posterior circulation, such as wide-neck basilar artery (BA) tip aneurysms, are not easy to access for direct intervention. ${ }^{3}$

Here, we report our experiences in the management of intracranial aneurysms associated with MMD at our institution during the past 10 years. We have focused on treatment strategies according to the type and location of the aneurysms.

\section{Methods}

The research ethics board of our institution approved the protocol for this prospective single-center study. Each patient or family member signed an informed consent for those who were enrolled in this study.

\section{Study Population}

Between January 2007 and March 2016, 1214 patients with MMD were treated at our institution. All patients had angiographically verified MMD under the guidelines of the Research Committee on MMD according to the Ministry of Health and Welfare in Japan. ${ }^{4}$ Among these MMD patients, 34 patients had intracranial aneurysms, with an incidence of 2.8\% (34/1214). There were 17 men and 17 women ranging in age from 11 to 67 years (mean 44.2 years). Clinical presentation included SAH in 8 patients, intraventricular hemorrhage (IVH) in 11, intracerebral hemorrhage (ICH) in 3, ICH with IVH in 9, SAH with ICH in 1, and ischemic stroke in 2. Among these patients, 2 harbored multiple aneurysms ( 2 aneurysms). Thus, there were 36 aneurysms in this cohort. Of these, 17 (47.2\%) were peripheral aneurysms and $19(52.8 \%)$ were main trunk aneurysms. The detailed locations and characteristics of the aneurysms are listed in Tables 1 and 2. All of these aneurysms were small (mean diameter $2.5 \mathrm{~mm}$, range $1-7 \mathrm{~mm}$ ) and saccular aneurysms.

\section{Treatment Strategy}

In peripheral aneurysms with an accessible endovascular approach, selective embolization of the lesion was performed, with preservation or distal occlusion of the parent artery and then treatment with cerebral revascularization.
TABLE 1. Locations of MMD-associated aneurysms

\begin{tabular}{|c|c|c|c|c|c|c|c|}
\hline \multirow[b]{2}{*}{ Location } & \multicolumn{6}{|c|}{ Manifestation } & \multirow[b]{2}{*}{ Total } \\
\hline & $\mathrm{SAH}$ & IVH & $\mathrm{ICH}$ & $\begin{array}{c}\mathrm{ICH} \\
+ \\
\mathrm{IVH}\end{array}$ & $\begin{array}{c}\mathrm{SAH} \\
+ \\
\mathrm{ICH}\end{array}$ & Ischemia & \\
\hline \multicolumn{8}{|c|}{ Peripheral aneurysms } \\
\hline Distal AChA & & 4 & & $2^{*}$ & & & 6 \\
\hline Distal PChA & & 5 & & 1 & & & 6 \\
\hline LSA & & & 1 & 1 & & & 2 \\
\hline Distal ACA & & & & 1 & & & 1 \\
\hline Distal MCA & & & & 1 & & & 1 \\
\hline Distal PCA & & & & 1 & & & 1 \\
\hline \multicolumn{8}{|c|}{ Main trunk aneurysms } \\
\hline $\mathrm{ACoA}$ & 1 & 1 & $2 \dagger$ & 1 & & 1 & 6 \\
\hline ACA & & & & 1 & & & 1 \\
\hline MCA & 1 & & 1 & & & & 2 \\
\hline ICA bifur & 1 & & & & & & 1 \\
\hline OphA & 1 & & & & & & 1 \\
\hline AChA & 1 & & & & & & 1 \\
\hline PCoA & 1 & & & & & & 1 \\
\hline BA & 1 & 1 & & & 1 & 1 & 4 \\
\hline PCA & 1 & & & 1 & & & 2 \\
\hline
\end{tabular}

Bifur = bifurcation; $\mathrm{PCOA}=$ posterior communicating artery.

* One of the aneurysms was found in a patient who harbored both distal AChA and PCA aneurysms and presented with ICH and IVH.

$\dagger$ One of the aneurysms was found in a patient who harbored both ACoA and LSA aneurysms and presented with ICH.

Aneurysms without an accessible endovascular route were treated only by revascularization. In main trunk aneurysms in the anterior circulation, clipping or coiling was performed, followed by simultaneous or staged revascularization. In main trunk aneurysms in the posterior circulation, coiling combined with revascularization was the first choice. Otherwise, revascularization alone was intended. The stent assistance technique was not used in this cohort for fear of the bleeding risk of antiplatelet therapy. Revascularization was performed in the more severe ischemic hemisphere in patients with ischemic types of

TABLE 2. Characteristics of MMD-associated aneurysms

\begin{tabular}{lrcc}
\hline Characteristic & Total & Peripheral Aneurysms & Main Trunk Aneurysms \\
\hline No. of lesions & 36 & 17 & 19 \\
\hline Size & & & \\
\hline$<3 \mathrm{~mm}$ & 22 & 16 & 12 \\
\hline $3-5 \mathrm{~mm}$ & 13 & 1 & 1 \\
\hline$>5 \mathrm{~mm}$ & 1 & 0 & \\
\hline Neck & & & 12 \\
\hline Broad & 7 & 0 & \\
\hline Narrow & 29 & 17 & 15 \\
\hline Morphology & & & 4 \\
\hline Regular & 29 & 14 & \\
\hline Irregular & 7 & 3 & \\
\hline
\end{tabular}




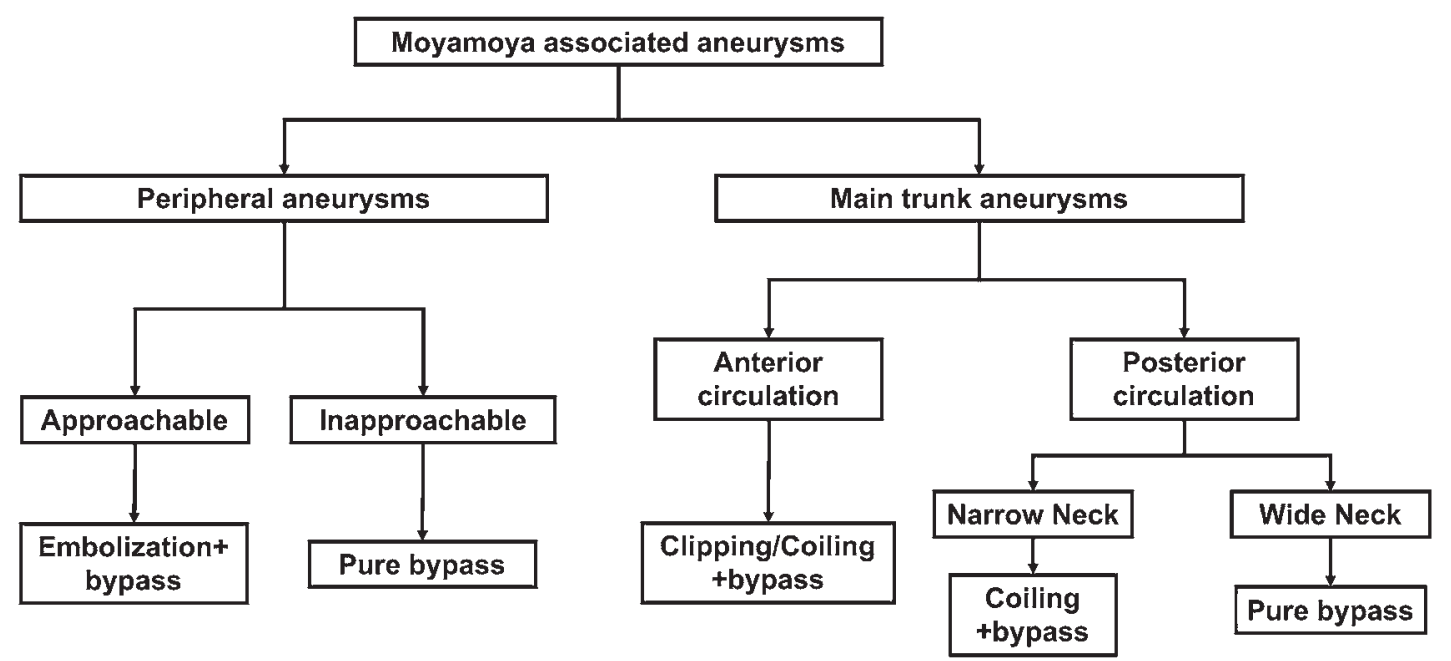

FIG. 1. Management algorithm for MMD-associated aneurysms.

lesions, or in the hemorrhagic hemisphere in those with hemorrhagic types (Fig. 1).

\section{Endovascular Procedure}

All procedures were performed using a uniplane angiographic system (Zeego, Siemens) with the patient under general anesthesia. Vascular access was obtained via a transfemoral approach by using a 5.0- or 6.0-Fr Envoy guiding catheter (Cordis). With the guidance of a roadmapping technique, a microcatheter (Echelon-14, Echelon-10, or Marathon; Covidien) was navigated to the aneurysm sac-or as close as possible to it-in the parent artery. Detachable coils (Guglielmi detachable coils, Stryker Neurovascular; Microplex, MicroVention) or Onyx 18 (Covidien) were used to embolize the aneurysm or the distal parent artery as close as possible to the lesion.

\section{Surgical Clipping}

Surgical clipping was performed simultaneously with cerebral revascularization. An enlarged modified pterional approach was used. Results of electrophysiological monitoring, including somatosensory evoked potentials and motor evoked potentials, were recorded in all cases. Angiographic intraoperative evaluation was performed using indocyanine green videoangiography.

\section{Cerebral Revascularization}

Revascularization procedures were performed as previously described. ${ }^{5,30}$ Briefly, the patient underwent a combined surgery containing direct bypass and indirect bypass. The direct procedure was superficial temporal artery-middle cerebral artery (STA-MCA) anastomosis. The indirect procedure was encephaloduromyosynangiosis (EDMS). After the direct bypass, indocyanine green fluorescence was regularly used to assess bypass patency.

\section{Clinical and Angiographic Follow-Up}

Neurological outcome was assessed using the modified Rankin Scale (mRS). All patients were advised to be clinically evaluated at 3,6, and 12 months after treatment, and yearly thereafter. An angiographic follow-up was scheduled at 6 months after the operation and then yearly thereafter for the assessment of the aneurysm and the effectiveness of the revascularization. The anastomosis was evaluated with reference to the criteria proposed by Matsushima and Inaba for the evaluation of transdural collateral vessels after revascularization: $\mathrm{A}=$ area perfused by the synangiosis is greater than two-thirds of the MCA territory; $\mathrm{B}=$ area perfused by the synangiosis is between one-third and two-thirds of the MCA territory; and C = area perfused by the synangiosis is less than one-third of the MCA territory. ${ }^{18,24}$ The Raymond classification scale was used for the postoperative angiographic result to evaluate the outcome of the aneurysm (see Roy et al. ${ }^{26}$ ).

\section{Results}

\section{Surgical Modalities}

Of the 17 peripheral aneurysms, 3 were treated with selective occlusion using coils. One aneurysm was treated with Onyx 18 to occlude the distal parent artery when the microcatheter approached but failed to reach the sac. The other 13 aneurysms were treated with cerebral revascularization alone, because of the inaccessible arterial approach to the aneurysms. Of the 13 main trunk aneurysms in the anterior circulation, 10 lesions were clipped with the assistance of intraoperative monitoring, followed by simultaneous cerebral revascularization, and the other 3 were treated with endovascular coiling followed by staged cerebral revascularization. Of the 6 main trunk aneurysms in the posterior circulation, 4 underwent endovascular coiling, whereas the remaining 2 were treated with pure revascularization. The details of the treatment modalities are listed in Table 3.

\section{Initial Results and Complications}

Immediate angiographic results in 11 aneurysms treated with the endovascular modality were evaluated using digital subtraction angiography (DSA) images. Complete occlusion was achieved in all aneurysms. In the 10 cases treated with microsurgical clipping, indocyanine green showed complete occlusion of the aneurysms. A com- 
TABLE 3. Treatment modality for MMD-associated aneurysms

\begin{tabular}{|c|c|c|c|c|c|c|c|c|c|}
\hline \multirow[b]{2}{*}{ Treatment Details } & \multicolumn{3}{|c|}{ Peripheral } & \multicolumn{3}{|c|}{ Anterior Main Trunk } & \multicolumn{3}{|c|}{ Posterior Main Trunk } \\
\hline & EVT + Rev & $M S+\operatorname{Rev}$ & Rev A & EVT + Rev & MS + Rev & Rev A & EVT + Rev & MS + Rev & Rev A \\
\hline Total no. treated & 4 & 0 & 13 & 3 & 10 & 0 & 4 & 0 & 2 \\
\hline \multicolumn{10}{|l|}{ Initial result } \\
\hline \multicolumn{10}{|l|}{ Aneurysm } \\
\hline Complete occlusion & 4 & 0 & 0 & 3 & 10 & 0 & 4 & 0 & 0 \\
\hline Nearly complete occlusion & 0 & 0 & 0 & 0 & 0 & 0 & 0 & 0 & 0 \\
\hline Incomplete occlusion & 0 & 0 & 0 & 0 & 0 & 0 & 0 & 0 & 0 \\
\hline \multicolumn{10}{|l|}{ Anastomosis } \\
\hline Patent & 4 & 0 & 13 & 3 & $9^{*}$ & 0 & 4 & 0 & $1^{*}$ \\
\hline Stenosis & 0 & 0 & 0 & 0 & 0 & 0 & 0 & 0 & 0 \\
\hline Occluded & 0 & 0 & 0 & 0 & 0 & 0 & 0 & 0 & 0 \\
\hline
\end{tabular}

$\mathrm{EVT}$ = endovascular treatment; $\mathrm{MS}$ = microsurgery; Rev = revascularization; Rev A = revascularization alone.

${ }^{*}$ Numbers of aneurysms do not match the totals because 2 patients received EDMS due to lack of a recipient artery for bypass.

bined bypass was achieved in 32 patients. Intraoperative indocyanine green fluorescence showed $100 \%$ patency of the anastomosis. Two patients underwent indirect bypass due to the lack of a recipient artery on the brain surface (Table 3).

There were 3 procedure-related complications in this cohort. One coil protruded into the ICA during the endovascular procedure in 1 patient who harbored an ophthalmic artery (OphA) aneurysm. However, no neurological symptoms developed in this patient. An intracerebral hematoma was discovered in 1 patient with an anterior communicating artery (ACoA) aneurysm 24 hours after aneurysm clipping and revascularization, and was evacuated immediately. The patient displayed moderate disability at discharge, improved to mild disability at the 6-month follow-up, and recovered to normal at the 72-month follow-up. One patient with an ICA bifurcation aneurysm suffered an ipsilateral basal ganglia infarction 72 hours after aneurysm clipping and EDMS. The patient still had moderate disability at the latest follow-up (Table 4).

\section{Clinical Outcome}

No patient died during hospital admission. Clinical follow-up was available in all the patients of this cohort at a mean of 64.2 months (range 14-96 months). One patient who harbored a basilar apex aneurysm and underwent revascularization alone died of contralateral ICH 9 months after surgery. Of the surviving 33 patients, none suffered recurrent intracranial hemorrhage, cerebral ischemia, or aneurysm rupture during the follow-up. Three patients had severe neurological deficits (mRS Score 3-5) due either to the brain damage caused by the initial ictus of intracranial hemorrhage or to a surgical complication. The remaining
30 patients attained good outcomes, with or without mild neurological deficits (mRS Score $0-2$ ).

\section{Angiographic Outcome}

\section{Efficacy of Bypass}

The latest postoperative angiograms were obtained for all the patients at a mean of 21.5 months (range 6-44 months). All the bypass grafts were confirmed patent, including for the patient who died. Transdural and transpial collaterals on the surgical site were graded according to the Matsushima criteria (Table 5).

\section{Aneurysm Obliteration}

Complete occlusion was achieved in all 21 aneurysms that were clipped or embolized. Of the remaining 15 aneurysms that were not directly treated, 12 of 13 peripheral aneurysms were obliterated during the follow-up, whereas 1 remained stable. One of 2 posterior main trunk aneurysms was stable, whereas the patient died of contralateral ICH as mentioned above; the other was smaller on the follow-up image (Table 6).

\section{Illustrative Cases}

\section{Case 7}

A 40-year-old man was admitted to our hospital with a severe explosive headache. A CT scan revealed SAH. An angiography study demonstrated MMD associated with a saccular aneurysm arising from the $\mathrm{P}_{1} / \mathrm{P}_{2}$ junction of the right posterior cerebral artery (PCA). The aneurysm was occluded by coil embolization immediately after DSA. Right STA-MCA bypass combined with EDMS was per-

TABLE 4. Procedure-related complications

\begin{tabular}{rcllllll}
\hline Case No. & Age (yrs), Sex & Presentation & Aneurysm & Treatment & Complication & Morbidity & Mortality \\
\hline 8 & $46, \mathrm{M}$ & SAH & OphA & EVT + Rev & Coil protrusion to parent artery & No & No \\
\hline 11 & $26, \mathrm{M}$ & Ischemia & ACoA & MS + Rev & Ipsilat intracerebral hematoma & No & No \\
\hline 18 & $46, \mathrm{M}$ & SAH & ICA bifur & MS + Rev & Ipsilat basal ganglia infarction & Moderate & No \\
\hline
\end{tabular}


Treatment for aneurysms in patients with MMD

TABLE 5. Angiographic efficacy of revascularization

\begin{tabular}{clc}
\hline $\begin{array}{c}\text { Matsushima } \\
\text { Grade }\end{array}$ & \multicolumn{1}{c}{ Description } & $\begin{array}{c}\text { No. of } \\
\text { Patients }\end{array}$ \\
\hline A & $\begin{array}{c}\text { Area perfused by the synangiosis is } \\
>2 / 3 \text { of the MCA territory }\end{array}$ & 26 \\
\hline B & $\begin{array}{c}\text { Area perfused by the synangiosis is } \\
\text { btwn } 1 / 3 \& 2 / 3 \text { of the MCA territory }\end{array}$ & 7 \\
\hline C & $\begin{array}{c}\text { Area perfused by the synangiosis is } \\
<1 / 3 \text { of the MCA territory }\end{array}$ & 1 \\
\hline
\end{tabular}

formed 1 month after SAH ictus. A follow-up angiography study obtained 16 months later showed complete obliteration of the aneurysm and a patent bypass stoma providing Grade A collateral distribution (Fig. 2).

\section{Case 14}

A 26-year-old woman suffered explosive headaches twice in 1 month. A CT scan revealed a pure IVH. An angiography study showed MMD associated with a peripheral aneurysm in the distal segment of the left anterior choroidal artery (AChA). In this case, a strategy of aneurysm or parent artery embolization was planned. A
TABLE 6. Angiographic outcome of indirectly treated aneurysms

\begin{tabular}{lcc}
\hline & \multicolumn{2}{c}{ Location } \\
\cline { 2 - 3 } Status & Peripheral & Posterior Main Trunk \\
\hline Ruptured & 0 & 0 \\
\hline Enlarged & 0 & 0 \\
\hline Stable & 1 & 1 \\
\hline Smaller & 0 & 1 \\
\hline Obliterated & 12 & 0 \\
\hline
\end{tabular}

Marathon microcatheter was placed in the distal AChA and was advanced as close as possible to the sac. A total of $0.1 \mathrm{ml}$ Onyx 18 was injected to occlude the aneurysm and its proximal parent artery. Left cerebral revascularization was performed 1 month later. The follow-up angiography study performed 8 months later showed complete occlusion of the aneurysm and Grade A collateral supply to the MCA territory after the bypass (Fig. 3).

\section{Case 19}

A 28-year-old woman reported the sudden onset of a severe headache. A CT scan showed bilateral IVH. An angiography study indicated MMD, with peripheral an-
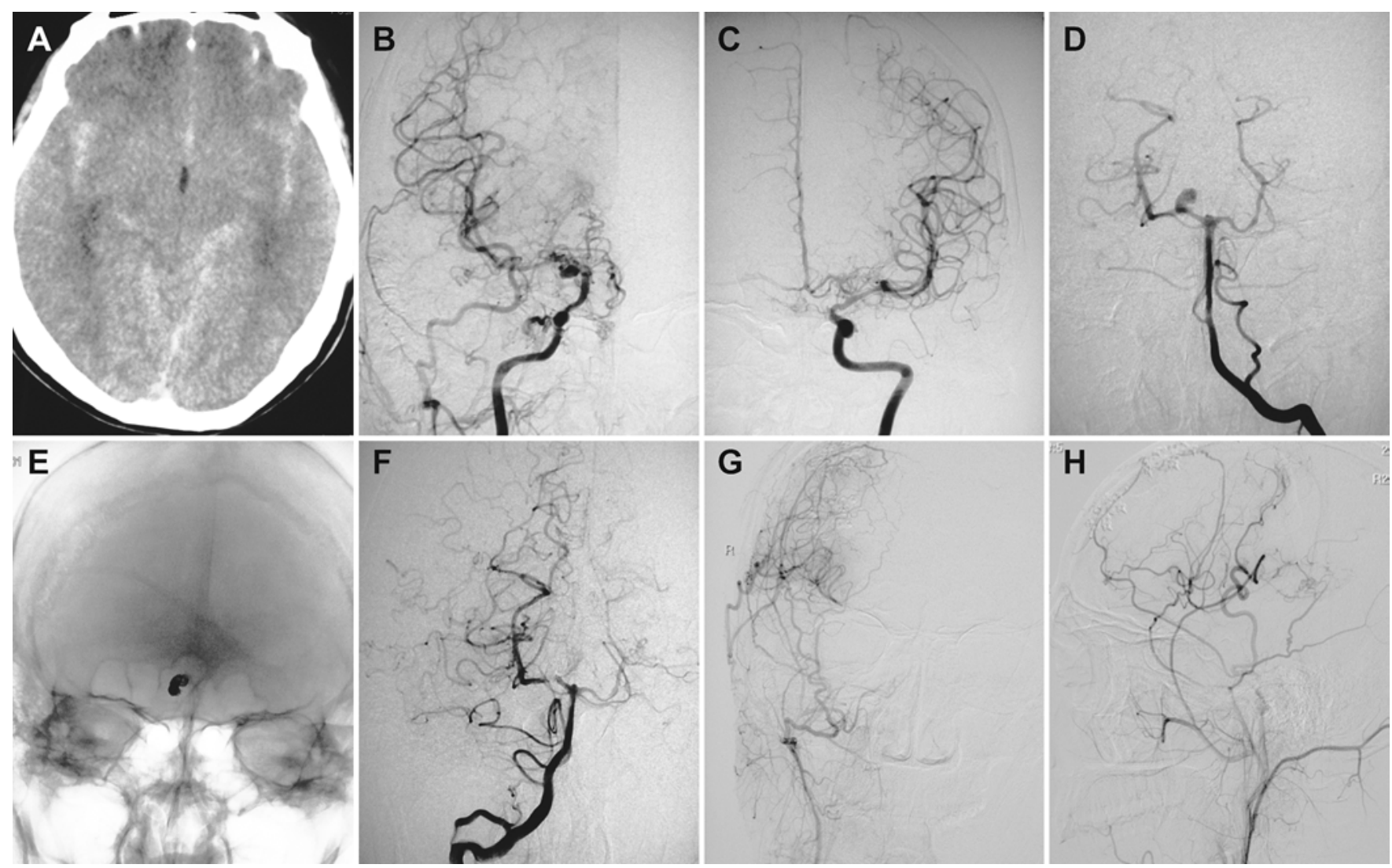

FIG. 2. Case 7. A: Axial CT scan revealing SAH in the longitudinal fissure, sylvian fissure, and quadrigeminal cistern. B and C: Bilateral ICA angiography studies showing MMD with Suzuki Stage III. D: Vertebral angiography study showing a saccular aneurysm at the $P_{1} / P_{2}$ junction of the right PCA. E and F: Follow-up vertebral angiography studies showing complete obliteration of the aneurysm. G and H: Follow-up external carotid artery (ECA) angiography studies showing Matsushima Grade A collateral compensation supplied by the anastomosis. 

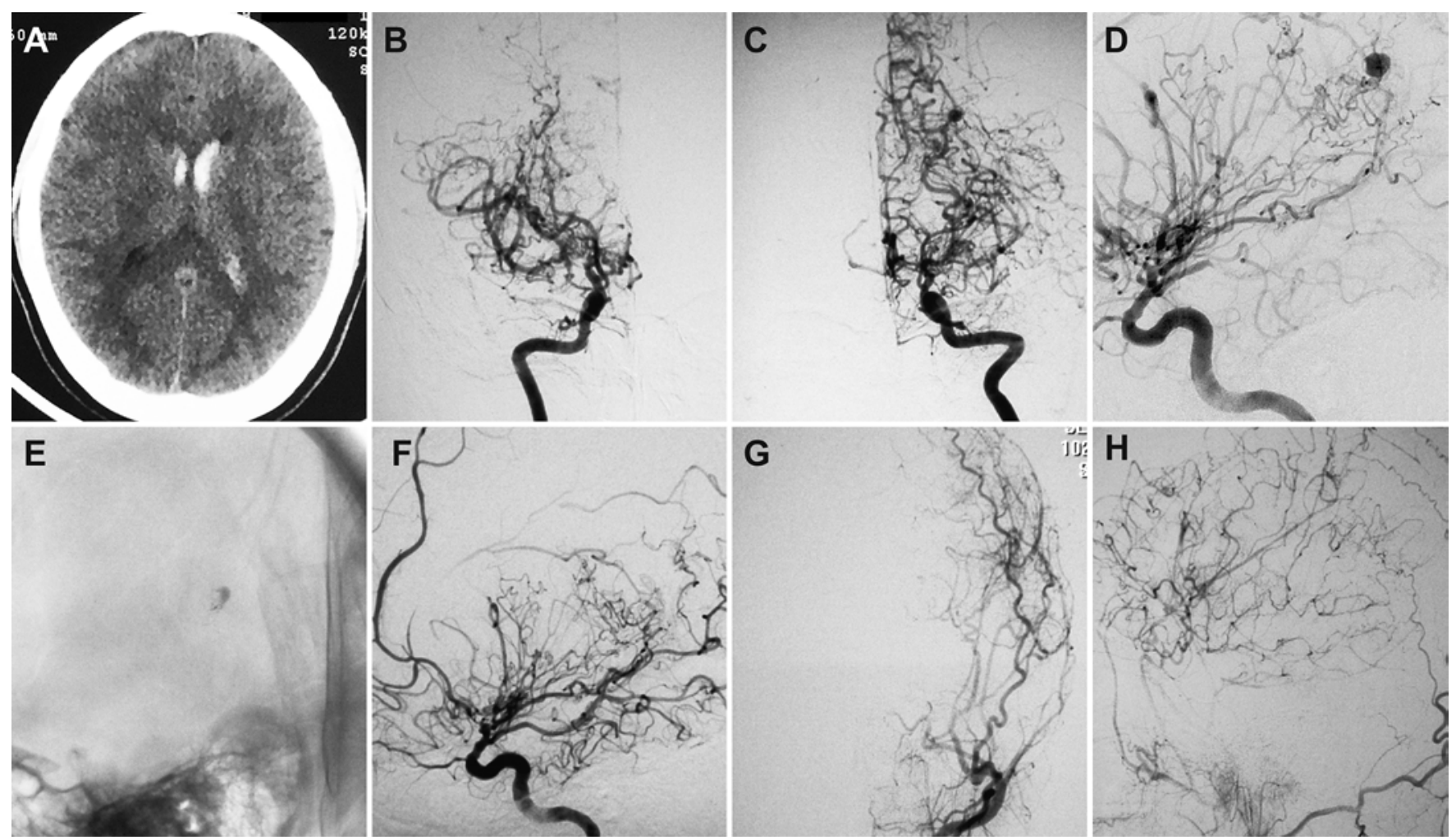

FIG. 3. Case 14. A: Axial CT scan revealing bilateral IVH. B-D: Bilateral ICA angiography studies showing MMD with a peripheral aneurysm in the distal segment of the left AChA. E and F: Follow-up ICA angiography studies showing complete obliteration of the aneurysm by Onyx 18. G and H: Follow-up ECA angiography studies showing Matsushima Grade A collateral compensation supplied by the anastomosis.

eurysms located in the distal segment of the right AChA. Because endovascular coiling was technically difficult or impossible due to the tortuosity and small diameter of the patent artery, pure cerebral revascularization was performed 2 months later when the hemorrhage was dissolved. The patient had no recurrent intracranial hemorrhage thereafter. Follow-up angiography performed 17 months after surgery showed total obliteration of the aneurysm and Grade A collateral vessels provided by the bypass procedure (Fig. 4).

\section{Case 29}

A 37-year-old woman presented with an explosive headache and consciousness disturbance. A CT scan showed right caudate hemorrhage and bilateral IVH. To relieve the intracranial pressure, external ventricular drainage was performed on an emergency basis. Angiography indicated MMD with a peripheral aneurysm in 1 lenticulostriate artery (LSA) from the right MCA. Because of the small size of the LSA, there was no accessible route to approach the aneurysm. Therefore, pure cerebral revascularization was performed 2 months later. A follow-up angiography sequence performed 6 months later showed total obliteration of the aneurysm and Grade B collateral compensation in the MCA territory (Fig. 5).

\section{Discussion}

An MMD-associated aneurysm is hazardous due to its high risk of rupture. Kawaguchi reviewed 111 cases of MMD-associated aneurysms and found 99 cases (89\%) that were manifested with intracranial hemorrhage..$^{13}$ In our series, 32 of 34 patients presented with various types of intracranial bleeding, indicating the high risk of bleeding of MMD-related aneurysms. Therefore, such aneurysms should be treated promptly and effectively. Although MMD-associated aneurysms have been frequently reported in the previous literature, the number of cases was relatively small and no single study provided enough information for developing an optimal treatment strategy for these aneurysms.$^{33}$ Recently, great advances have been achieved in the field of microsurgery and interventional radiology, but there is still no consensus on the treatment strategy for MMD-associated aneurysms.

Generally, MMD-related aneurysms are prone to occur on the main trunk of the circle of Willis and peripheral vessels, such as the moyamoya vessels (LSA and distal anterior cerebral artery [ACA]/MCA/PCA) and the collateral vessels (AChA and posterior choroidal artery [PChA]). Because aneurysms at different sites differ in pathogenesis and hemodynamic features, the planning of their treatment strategy should be facilitated based on the locations.

Choroidal artery (12 aneurysms) or LSA ( 2 aneurysms) are the most common locations for peripheral aneurysms. As shown in our series, all the patients who harbored such aneurysms presented with ICH or IVH. These aneurysms were believed to be the direct cause of bleeding in MMD. The real pathology of peripheral aneurysms is controver- 

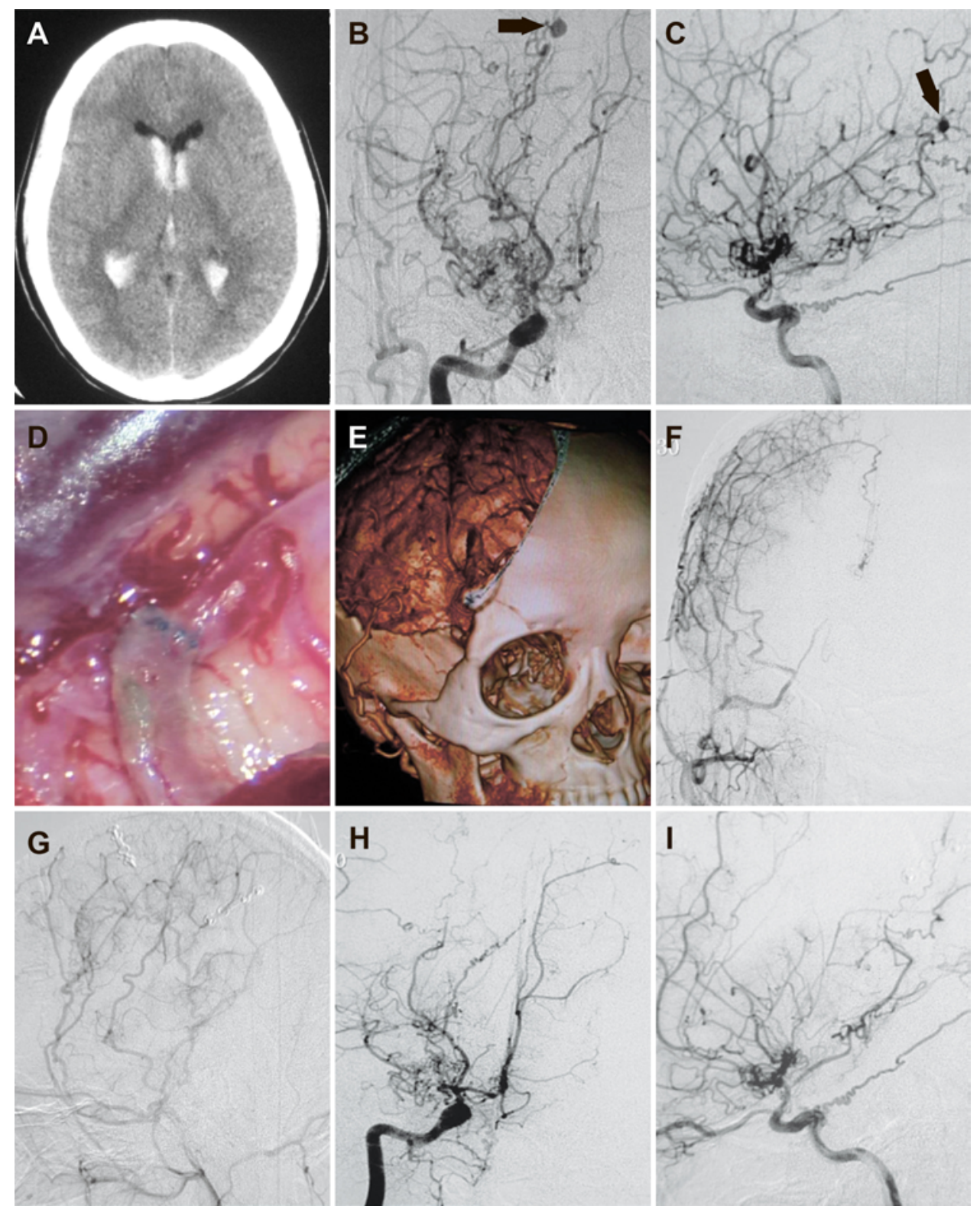

FIG. 4. Case 19. A: Axial CT scan revealing bilateral IVH. B and C: ICA angiography studies showing MMD with a peripheral aneurysm in the distal segment in the right AChA (arrows). D: Intraoperative photograph showing the anastomosis between the STA and the cortical branch of the MCA. E: A 3D CT angiography study showing the patency of the bypass. F and G: Follow-up ECA angiography studies showing Matsushima Grade A collateral compensation supplied by the anastomosis. $\mathrm{H}$ and I: Follow-up ICA angiography studies showing complete obliteration of the aneurysm. Figure is available in color online only.

sial. Some authors believed these aneurysms were true aneurysms with a stable status. ${ }^{16,21}$ Others considered these as pseudoaneurysms with a tendency for early increase in size. ${ }^{6,22,32}$ Therefore, it is often considered necessary to treat these aneurysms with surgical interventions. Direct surgery such as aneurysmectomy or neck clipping could be effective, but has a high risk due to the deep location of these aneurysms; fragile moyamoya vessels and perforators may hinder the approach,,$^{20}$ and the distal location increases the difficulty of localization. Hence, surgical clipping is not recommended in these patients.

Endovascular treatment is less invasive and should be the first choice to treat these peripheral aneurysms if there is an accessible approach for the microcatheter. As shown in our series, if available, selective endovascular coiling or Onyx injection showed a $100 \%$ obliteration rate on images obtained immediately postoperatively and/or on follow-up images. However, because of the distal location of the peripheral aneurysms, the application of endovascular treatment is limited if the parent artery is tortuous. Revascularization has proved to be effective in previously reported cases. ${ }^{7,17,22}$ Many studies have demonstrated that hemodynamic stress on the fragile MMD vessels or dilated choroidal arteries might be correlated with the hemorrhagic ictus and peripheral aneurysm formation. ${ }^{2,9,10,15,28}$ Bypass surgery can develop additional collateral flow in the surgical territory and 

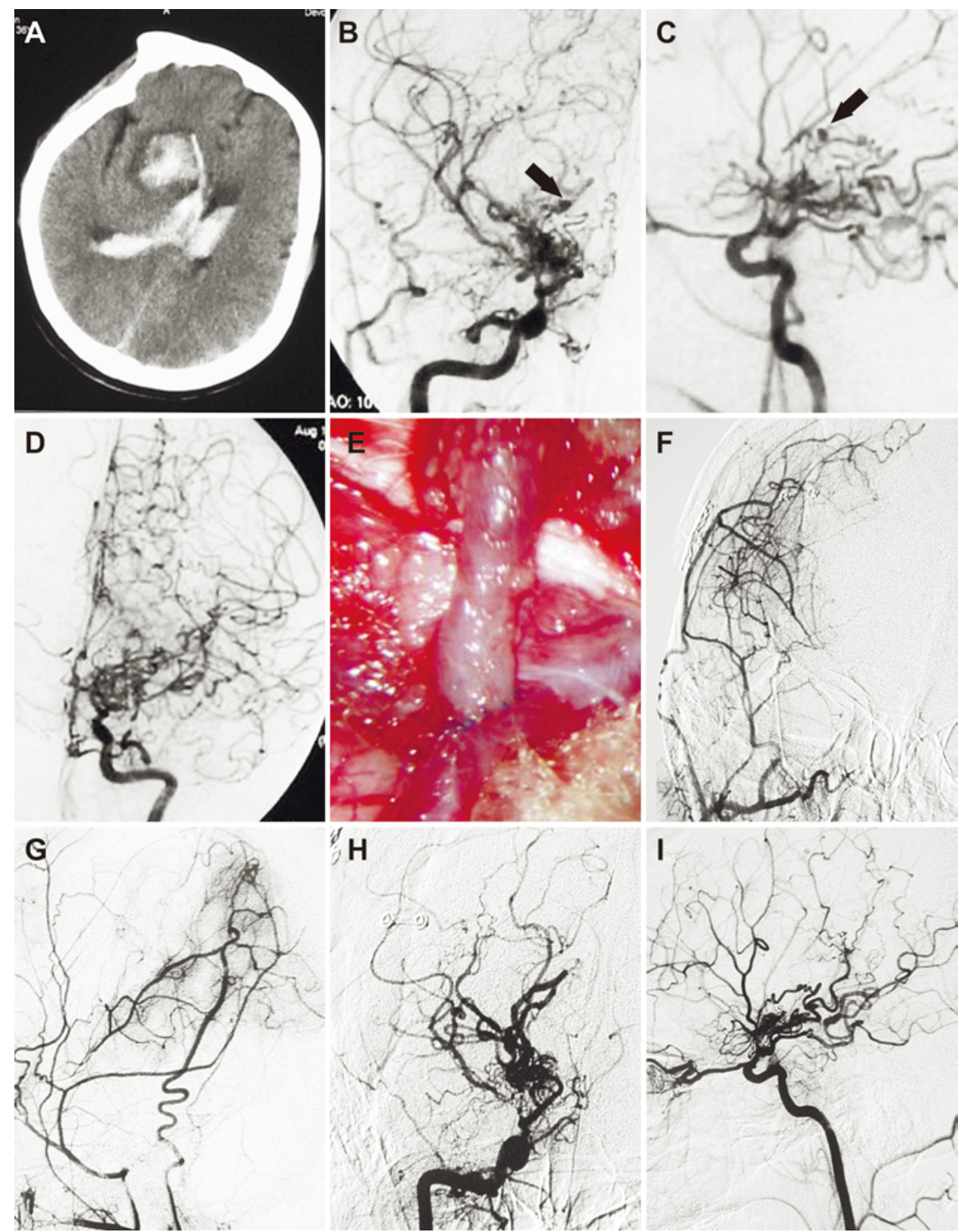

FIG. 5. Case 29. A: Axial CT scan revealing a right caudate hematoma with bilateral IVH. B-D: Bilateral ICA angiography studies showing MMD with peripheral aneurysm in 1 LSA originating from the right MCA (arrows). E: Intraoperative photograph showing the anastomosis between the STA and cortical branch of the MCA. F and G: Follow-up ECA angiography studies showing Matsushima Grade B collateral compensation supplied by the anastomosis. H and I: Follow-up ICA angiography studies showing complete obliteration of the aneurysm. Figure is available in color online only.

decrease the hemodynamic stress on the pathological moyamoya vessels or delicate choroidal arteries, thus shrinking the distal aneurysms. In our patients, we achieved a high obliteration rate $(92.3 \%, 12 / 13)$ of peripheral aneurysms treated with revascularization alone. This result strengthened the relationship between the hemodynamic change and the fate of the peripheral aneurysms in MMD.

Main trunk aneurysms are predominantly located in the circle of Willis. ${ }^{3}$ In the cases we report here, the most common locations for main trunk aneurysms were in the ACoA (6 aneurysms), followed by the BA tip (4 aneurysms). These aneurysms were more likely to be cor- related with outflow resistance in the distal ICA that was causing persistent high-flow stress on the anterior circulation and blood compensation burden on the vertebrobasilar system..$^{19}$ Most main trunk aneurysms are true saccular aneurysms with a high rupture risk. In our series, 14 of 19 main trunk aneurysms caused SAH or IVH. Thus, these aneurysms should be treated with effective modalities. The medical literature on the surgical management of MMD-associated main trunk aneurysms is limited to case reports and small series, the results of which suggest that direct surgical intervention generally results in good outcomes. ${ }^{33}$ For aneurysms in the anterior circulation, some 
literature has suggested that these may spontaneously disappear with the progression of MMD due to the gradual steno-occlusion of the distal ICA and proximal ACA and MCA. ${ }^{24,28}$ However, such conservative observation may last for a long period and carry a risk of rebleeding, especially for ruptured aneurysms. Therefore, direct clipping seems to be the ideal treatment if revascularization can be achieved in the same craniotomy.

Endovascular coiling is considered an alternative treatment for anterior circulation aneurysms. However, we do not advocate the stent-assisted technique, considering the increased risk for revascularization caused by antiplatelet therapy after stent placement. For posterior circulation aneurysms, because direct clipping is difficult and hazardous, $1,13,14$ embolization with coils has become the preferred treatment. However for broad-neck aneurysms, simple coiling is challenging because of the instability of coils and the initial low complete occlusion rate. ${ }^{8}$ The stent-assisted technique is also avoided for fear of the increased bleeding risk at revascularization. On the basis of the theory of hemodynamic alteration after revascularization, we attempted to treat some main trunk aneurysms by revascularization alone if stent-free coiling was not available. As shown in our data, 1 aneurysm located in the BA tip became smaller; the other remained stable during the follow-up period. None of these aneurysms ruptured or reruptured, indicating that revascularization alone might be an alternative option for main trunk aneurysms in the posterior circulation. The case of aneurysm shrinkage allowed us to hypothesize that revascularization may relieve the burden on the vertebrobasilar system by reconstructing the anterior circulation, thereby reducing the risk of aneurysm enlargement or bleeding. However, we must admit that a longer DSA follow-up time and a larger series of cases are needed to support our hypothesis.

The current study has several limitations. First, although to our knowledge this was the largest reported series of patients with MMD-associated aneurysms in a single center, the case number was relatively limited. Second, this is not a randomized controlled study. There may have been bias in patient selection and demographics.

\section{Conclusions}

Our results indicate that our current treatment strategy may provide a benefit for patients with MMD-associated aneurysms. However, its long-term efficacy should be confirmed in a larger series of cases.

\section{Acknowledgments}

This study was supported by Grant 81500987 from the National Natural Science Foundation of China (NSFC) and Grant 15140902300 from the Science and Technology Commission of Shanghai Municipality (STCSM). The content is solely the responsibility of the authors and does not necessarily represent the official views of the NSFC and STCSM.

\section{References}

1. Bhattacharjee AK, Tamaki N, Minami H, Ehara K: Moyamoya disease associated with basilar tip aneurysm. J Clin Neurosci 6:268-271, 1999

2. Burke GM, Burke AM, Sherma AK, Hurley MC, Batjer HH,
Bendok BR: Moyamoya disease: a summary. Neurosurg Focus 26(4):E11, 2009

3. Chen Y, Dai D, Fang Y, Yang P, Huang Q, Zhao W, et al: Endovascular treatment of ruptured large or wide-neck basilar tip aneurysms associated with moyamoya disease using the stent-assisted coil technique. J Stroke Cerebrovasc Dis 24:2229-2235, 2015

4. Fukui M: Guidelines for the diagnosis and treatment of spontaneous occlusion of the circle of Willis ('moyamoya' disease). Research Committee on Spontaneous Occlusion of the Circle of Willis (Moyamoya Disease) of the Ministry of Health and Welfare, Japan. Clin Neurol Neurosurg 99 (Suppl 2):S238-S240, 1997

5. Gu Y, Ni W, Jiang H, Ning G, Xu B, Tian Y, et al: Efficacy of extracranial-intracranial revascularization for non-moyamoya steno-occlusive cerebrovascular disease in a series of 66 patients. J Clin Neurosci 19:1408-1415, 2012

6. Hamada J, Hashimoto N, Tsukahara T: Moyamoya disease with repeated intraventricular hemorrhage due to aneurysm rupture. Report of two cases. J Neurosurg 80:328-331, 1994

7. He K, Zhu W, Chen L, Mao Y: Management of distal choroidal artery aneurysms in patients with moyamoya disease: report of three cases and review of the literature. World $\mathbf{J}$ Surg Oncol 11:187, 2013

8. Henkes H, Fischer S, Mariushi W, Weber W, Liebig T, Miloslavski E, et al: Angiographic and clinical results in 316 coiltreated basilar artery bifurcation aneurysms. J Neurosurg 103:990-999, 2005

9. Irikura K, Miyasaka Y, Kurata A, Tanaka R, Yamada M, Kan S, et al: The effect of encephalo-myo-synangiosis on abnormal collateral vessels in childhood moyamoya disease. Neurol Res 22:341-346, 2000

10. Iwama T, Hashimoto N, Tsukahara T, Miyake H: Superficial temporal artery to anterior cerebral artery direct anastomosis in patients with moyamoya disease. Clin Neurol Neurosurg 99 (Suppl 2):S134-S136, 1997

11. Iwama T, Morimoto M, Hashimoto N, Goto Y, Todaka T, Sawada M: Mechanism of intracranial rebleeding in moyamoya disease. Clin Neurol Neurosurg 99 (Suppl 2):S187S190, 1997

12. Iwama T, Todaka T, Hashimoto N: Direct surgery for major artery aneurysm associated with moyamoya disease. Clin Neurol Neurosurg 99 (Suppl 2):S191-S193, 1997

13. Kawaguchi S, Sakaki T, Morimoto T, Kakizaki T, Kamada $\mathrm{K}$ : Characteristics of intracranial aneurysms associated with moyamoya disease. A review of 111 cases. Acta Neurochir (Wien) 138:1287-1294, 1996

14. Kodama N, Sato M, Sasaki T: Treatment of ruptured cerebral aneurysm in moyamoya disease. Surg Neurol 46:62-66, 1996

15. Kuroda S, Houkin K: Moyamoya disease: current concepts and future perspectives. Lancet Neurol 7:1056-1066, 2008

16. Lee JK, Lee JH, Kim SH, Lee MC: Distal anterior choroidal artery aneurysm in a patient with moyamoya disease: case report. Neurosurgery 48:222-225, 2001

17. Liu P, Lv XL, Liu AH, Chen C, Ge HJ, Jin HW, et al: Intracranial aneurysms associated with moyamoya disease in children: clinical features and long-term surgical outcome. World Neurosurg 94:513-520, 2016

18. Matsushima Y, Inaba Y: Moyamoya disease in children and its surgical treatment. Introduction of a new surgical procedure and its follow-up angiogram. Childs Brain 11:155-170, 1984

19. Morioka M, Hamada J, Todaka T, Yano S, Kai Y, Ushio Y: High-risk age for rebleeding in patients with hemorrhagic moyamoya disease: long-term follow-up study. Neurosurgery 52:1049-1055, 2003

20. Nagamine Y, Takahashi S, Sonobe M: Multiple intracranial aneurysms associated with moyamoya disease. Case report. J Neurosurg 54:673-676, 1981 
21. Nakai H, Yamamoto K, Sako K, Tanikawa R, Kunimoto M, Hashimoto M, et al: [A ruptured aneurysm at the peripheral collateral circulation of the anterior choroidal artery in a patient with moyamoya disease: a case report.] No Shinkei Geka 20:985-990, 1992 (Jpn)

22. Ni W, Xu F, Xu B, Liao Y, Gu Y, Song D: Disappearance of aneurysms associated with moyamoya disease after STAMCA anastomosis with encephaloduro myosynangiosis. J Clin Neurosci 19:485-487, 2012

23. Otawara Y, Ogasawara K, Seki K, Kibe M, Kubo Y, Ogawa A: Intracerebral hemorrhage after prophylactic revascularization in a patient with adult moyamoya disease. Surg Neurol 68:335-337, 2007

24. Peltier J, Vinchon M, Soto-Ares G, Dhellemmes P: Disappearance of a middle cerebral artery aneurysm associated with Moyamoya syndrome after revascularization in a child: case report. Childs Nerv Syst 24:1483-1487, 2008

25. Robertson RL, Burrows PE, Barnes PD, Robson CD, Poussaint TY, Scott RM: Angiographic changes after pial synangiosis in childhood moyamoya disease. AJNR Am J Neuroradiol 18:837-845, 1997

26. Roy D, Milot G, Raymond J: Endovascular treatment of unruptured aneurysms. Stroke 32:1998-2004, 2001

27. Sadatoh A, Yonekawa Y, Morooka Y, Imakita T: [A case of moyamoya disease with repeated intraventricular hemorrhage due to a ruptured pseudoaneurysm.] No Shinkei Geka 17:755-758, 1989 (Jpn)

28. Satoh T, Yamamoto Y, Asari S, Sakurai M, Suzuki K: Disappearance and development of cerebral aneurysms in moyamoya disease. Case report. J Neurosurg 58:949-953, 1983

29. Takahashi JC, Miyamoto S: Moyamoya disease: recent progress and outlook. Neurol Med Chir (Tokyo) 50:824-832, 2010

30. Xu B, Song DL, Mao Y, Gu YX, Xu H, Liao YJ, et al: Superficial temporal artery-middle cerebral artery bypass com- bined with encephalo-duro-myo-synangiosis in treating moyamoya disease: surgical techniques, indications and midterm follow-up results. Chin Med J (Engl) 125:4398-4405, 2012

31. Yeon JY, Kim JS, Hong SC: Incidental major artery aneurysms in patients with non-hemorrhagic moyamoya disease. Acta Neurochir (Wien) 153:1263-1270, 2011

32. Yuasa H, Tokito S, Izumi K, Hirabayashi K: Cerebrovascular moyamoya disease associated with an intracranial pseudoaneurysm. Case report. J Neurosurg 56:131-134, 1982

33. Zhang L, Xu K, Zhang Y, Wang X, Yu J: Treatment strategies for aneurysms associated with moyamoya disease. Int J Med Sci 12:234-242, 2015

\section{Disclosures}

The authors report no conflict of interest concerning the materials or methods used in this study or the findings specified in this paper.

\section{Author Contributions}

Conception and design: Gu, Ni, Jiang. Acquisition of data: Ni, Jiang, Yang, Su. Analysis and interpretation of data: Ni, Jiang, Lei, Yang, Su. Drafting the article: Ni, Lei, Yang, Su. Critically revising the article: $\mathrm{Gu}, \mathrm{Ni}$. Reviewed submitted version of manuscript: $\mathrm{Gu}, \mathrm{Ni}$, Jiang, Mao. Approved the final version of the manuscript on behalf of all authors: Gu. Statistical analysis: $\mathrm{Ni}$, Jiang, Lei. Administrative/technical/material support: Gu, Xu. Study supervision: Gu, Mao.

\section{Correspondence}

Yuxiang Gu, Department of Neurosurgery, Huashan Hospital, Fudan University, No. 12 Mid Wulumuqi Rd., Shanghai 200040, P. R. China. email: guyuxiang1972@126.com. 